seen again, when she walked well without a stick and no defect was noticeable in her gait.

Remarks by Mr. LockwooD.-It would hardly be right to draw any conclusions from this case as to the value of the serum which was given. I had not the opportunity of seeing the case when the joints were incised, or of seeing specimens of the pus. The fact that nothing grew in the cultures must not be taken to be evidence of the absence of streptococci. At times these organisms are exceedingly difficult to cultivate, although known to be present. Nevertheless, in this instance, some entirely different microbe may have caused the suppuration. The point of interest is the wonderfully complete recovery of function in the suppurating joints. This is due to the prompt treatment which the house surgeon, Mr. Maxwell, applied, and to the care which was afterwards taken to avoid any fresh infection from the wounds. Perhaps I may add that antistreptococcic serum was quite unavailing in a case of pyæmia which I saw with Dr. Samuel West and Dr. J. A. Smith, although streptococci were seen in the pus.

\section{CANNING TOWN MEDICAL MISSION} HOSPITAL.

A CASE OF ULCERATIVE ENDOCARDITIS TREATED WITH ANTISTREPTOCOCCIC SERUM.

(Under the care of Dr. Margaret Pearse.)

THE ordinary medicinal treatment of ulcerative endocarditis is so extremely unsatisfactory, and the rarity of recovery is so great, that any method which offers better prospects of cure is sure of a careful and extended trial. Though for years the causal connexion of streptococci and staphylococci with the disease has been recognised, it is only recently, since the introduction of serum therapy, that any hopes have arisen of combating successfully this most deadly disease. The use of antistreptococcic serum is still on its trial, and therefore every case in which it is employed should be put on record to assist in the formation of an opinion as to the value of the treatment. A case very similar to the following was published in THE LANCET last autumn. ${ }^{1}$

A girl, aged sixteen years, attended as an out-patient on Nov. 27 hb, 1896, complaining of having "had a cold" for three weeks and of her "breath being tight." There was marked dyspncea, the pulse and respiration rates were increased, and the temperature was $101^{\circ} \mathrm{F}$. Examination revealed rapid and exaggerated cardiac action, a loud mitral systolic murmur, and a faint diastolic murmur at the aortic area. The patient was put on salicylates and treated at home for a week. The temperature remained high, one evening rising to $105^{\circ}$. No improvement taking place she was admitted into the hospital on Dec. 4th. The history of the case obtained after admission was as follows. She had felt quite well till the commencement of November, when she "caught a bad cold that left ber chest choked up." She kept at her work, though feeling ill, till Nov. 23rd, when she complained of aching pains in the left shoulder, and on the $25 \mathrm{th}$ of palpitation and shortness of breath. On the $27 \mathrm{th}$ she visited the out-patient department for advice. No previous history of rheumatism was obtainable, or of ary rheumatic manifestations beyond a transient erythema a year before. On admission the patient did not seem to be very ill, being bright and lively. There was considerable dyspncea, but she was able to lie down. She complained of palpitation and of slight pain in the left shoulder. There was considerable perspiration without acid odour. No nodules, no rash, and no anæmia were present. On examining the heart the apex beat was seen and felt in the fifth interspace, three inches from mid-sternum. The area of cardiac dulness was increased to the right. The sounds were muffled at the apex, but a mitral systolic murmur was distinguisbable, as well as a diastolic aortic murmur, and some pericardial friction sounds. At the base of the right lung dulness on percussion and diminished breath-sounds indicated a small effusion. During the first week after admission, from Dec. 4th to 10 th, the patient showed signs of marked general improvement. The dyspnœea lessened, the slight joint pains disappeared, and the temperature gradually fell from $103^{\circ}$ to $100.4^{\circ}$ in the evening, and reached the normal in the morning. The pulserate fell from 114 to 84 and the respirations from 36 to 24 .
Some progression was apparent in the physical signs, however, indicating especially increase of tluid in the pericardium. The apex beat was indistinctly felt in the fourth interspace, the area of cardiac dulness extended up to the second interspace, and the sounds at the apex became more moffled. A systolic aortic murmur was heard as well as the diastolic aortic murmur. The edge of the spleen was indefinitely felt below the costal margin. The patient during this time was at first taking ten-grain doses of salicylate of soda every six hours, and then every four hours. During the second week, from Dec. 11th to 17th, the general condition of the patient remained much the same, except that the temperature again gradually rose to reach $102^{\circ}$ in the evening and an erythematous rash appeared which by degrees spread over the limbs and face, disappearing after three days. The fluid in the pericardium and at the right base diminished slightly in amount. The urine was normal, and continued so throughout the whole illness. During the third week, from Dec. 18th to $24 \mathrm{th}$, she did not complain of feeling more ill, but the temperature now remained persistently between $101^{\circ}$ and $103^{\circ}$, and the pulserate again rose to 116 . She complained of tenderness on palpation of the spleen. The physical signs remained much the same, but for again indicating slight increase of fluid in the pericardium. As the salicylate treatment seemed to be useless the patient was given five grains of salol twice a day, but the treatment had to be discontinued after two days on account of the severe abdominal pains felt immediately after each dose. On the 24th she was seen in consultation by Dr. Schorstein, and as the increasing severity of the symptoms suggested the probability of the endocardial mischief having assumed a malignant type, it was decided to try the effect of treatment by antistreptococcic serum. An abstract of the daily notes is as follows, and the accompanying chart indicates the wide range of the temperature.

The serum used was obtained from the British Institute of Preventive Medicine, and the injections, with two exceptions, were made under the skin of the abdomen. All other drugs were discontinued. On Dec. 26th the patient complained of pain over the præcordium. Many fresh friction sounds were heard. The area of splenic dulness was increased downwards and forwards, and there was tenderness on palpation. At 6 P.M. $2 \frac{1}{2}$ c.c. of antistreptococcic serum were injected. She felt no discomfort either generally or locally afterwards. During the evening sharp pleuritic pains were felt in the left axilla, and many friction sounds were heard. On the 27 th all pains had disappeared. At 8.30 P.M. 5 c.c. of the serum were injected. On the $28 \mathrm{th}$ the patient was much the same. At 6 P.M. 10 c.c. were injected. On the 29 th her general condition was good, though the temperature remained high, and there was no improvement in the physical signs. There was still a considerable amount of fluid in the pericardium, and some $\epsilon$ ffusion at the right base as well as the recent pleurisy on the left. On the 30th her general condition was not so good. She slept during the greater part of the day, but complained of pain over the præcordium and of very sharp plearitic pains in the left side. Many friction sounds were heard. At 11 A M. 20 c.c. were injected. There was no discomfort. On the 3lst the temperature had made a decided drop, falling to $100^{\circ}$, and at 11 A.M. 10 c.c. were injected. On Jan. Ist, 1897, there was an erythematous blush over the abdomen at the site of the last three punctures. Some improvement had taken place in the pbysical signs; the upper limit of the cardiac dulness was half an inch farther down, and there was less pericardial friction. The breath sounds were louder at the right base. Dulness and bronchial breatining at the angle of the left scapula and many friction sounds in the axilla were noted. On the 2 nd the rash over the abdomen had not faded, and a rash showed itself on the limbs and face similar to that which appeared before. It was much more raised than that upon the abdomen which followed the injections, and was very irritable. On auscultation the second sound at the apex was found to be reduplicated. On the $3 r d$ the temperature fell for the first time to normal. The injections were resumed after having been omitted for two days on account of the rash. At 10 A.M. 20 c.c. were injected, and within four hours the temperature rose to almost $104^{\circ}$. On the 4 th there was marked general improve ment in the patient. Her appetite was increasing. She felt no pains except a little at the site of the previous puncture in the gluteal region. On the 5 th the temperature was normal during the greater part of the day, and did not rise beyond $1016^{\circ}$. On the 6th the rashes had almost disappeared. The patient seemed to be decidedly stronger. There was marked. 
improvement in the physical signs. The apex beat was now The temperature persistently rose and failed to be accounted in the fifth interspace and the sounds at the apex were much for by the appearance of fresh rheumatic manifestaless muffled. Much pericardial friction was heard. The tions (beyond an erythema), or sufficiently increased severity breath sounds were distinct to the base of the right lung and of those already present, except as regards the endocarditis. the patch of bronchial breathing left had disappeared, but Here, as the notes show, soon after the patient's admission into some friction and moist sounds were still heard. At 4 P.M. hospital a systolic aortic murmur gradually appeared, amd 10 c.c. were injected. On the 7th there was a slight rise of later a presystolic mitral murmur, which afterwards gradually temperature in the evening and the pulse and respiration disappeared as the serum treatment was persisted in. This rates increased. During the night the patient was disturbed by uncontrollable cough; there was no expectoration. On the 8th the temperature fell below normal and the patient perspired profusely. At 10 A.M. 10 c.c. were injected and much pain at the site of puncture was complained of afterwards. Between 10 and 12 P.M. the temperature rose to $105^{\circ}$ and the cough again became troublesome. On the 9th there was further improvement in the physical signs. The area of cardiac dulness was practically normal, except that the right limit was at the right sternal border. The apex beat was in the fifth interspace three inches from mid-sternum. The sounds at the apex were no longer muffled, and a faint marked alteration in the murmurs, coupled with the high temperature of a hectic type and increase in the size of the spleen, led to the conclusion that the endocarditis was of the ulcerative rather than the simple type. Unfortunately, a bacteriological examination of the blood was only made on Jan. 11th, when, as the report said, "the case having been under treatment so long it was not surprising that. no streptococci were found." The verification of the diagnosis by the appearance of infarcts was fortunately averted by putting the patient under the serum treatment immediately the diagnosis' seemed in the least warrantable. The possi-
bility of typhoid fever was readily excluded by absence of all

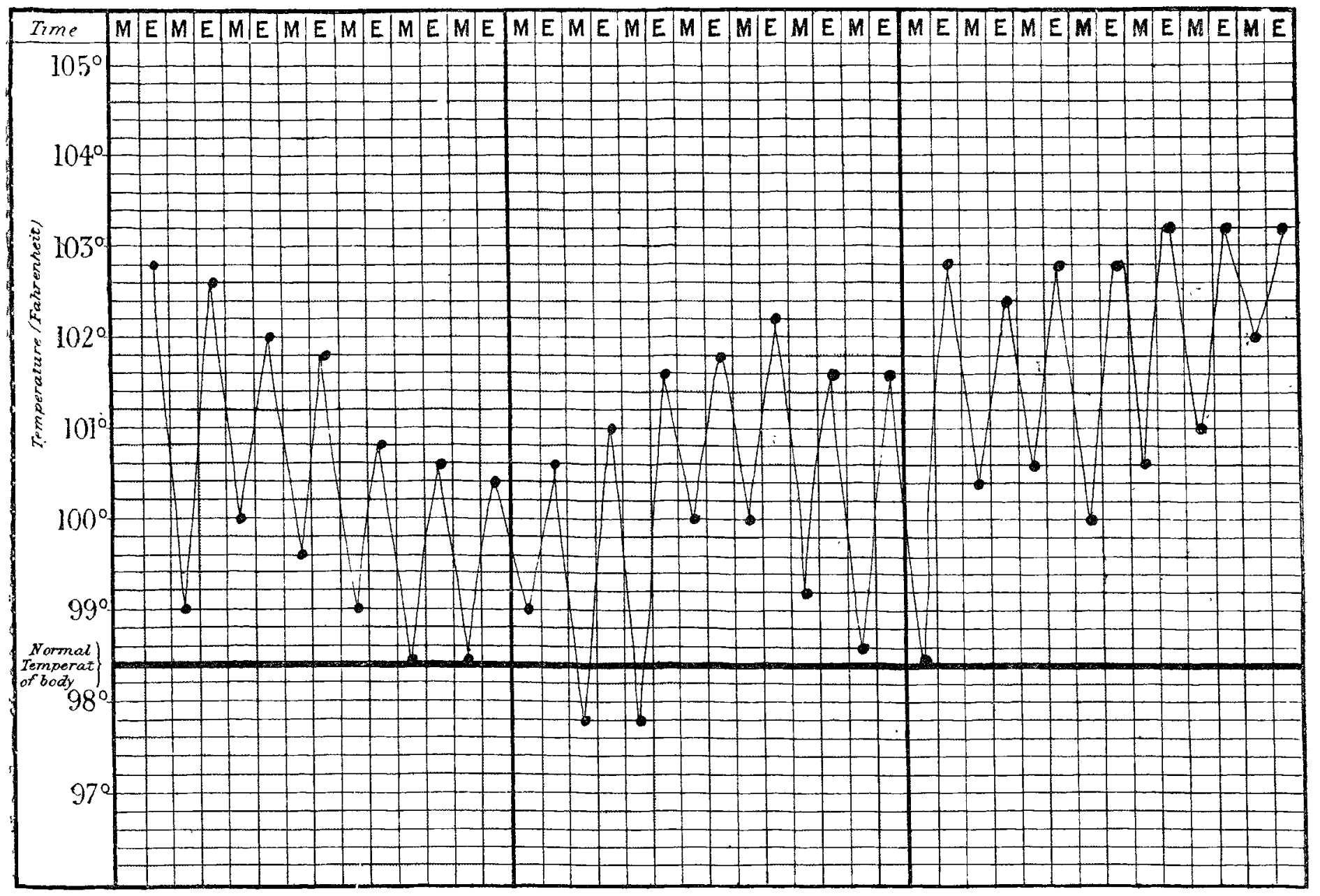

presystolic murmur was distinguishable. On the 11th the patient was very well and felt no pains. The temperature remained subnormal all day and did not again rise above normal. On the 23 rd the patient was practically well and said she felt quite strong. There was no dyspncea or palpitation. On examination all that was found was that the cardiac dulness still extended to the right border of the sternum, and that a double aortic and a mitral systolic murmur were present. The presystolic mitral murmur had quite disappeared. The patient was allowed to get up on this date, and on Feb. 5th she was discharged perfectly well

Remarks by Dr. MARGABET PEARSE.-The foregoing account speaks for itself of the value of having adopted the serum treatment. Salicylates, after producing temporary improvement, proved useless. Salol could not be borne, and the case assumed an increasingly serious aspect until the injections were commenced and persevered with, for improvement even then was slow and gradu 3 , though ultimately ending in perfect recovery. As to the diagnosis of the case it was difficult to arrive at any other. The illness was distinctly rheumatic in origin, as evidenced by the joint pains and the implications of both pericardium and endocardium. With the diminution of joint pains temporary improvement occurred, but this was soon replaced by progressive increase in the seriousness of the patient's condition. the characteristic symptoms except high temperature and slight increase in the size of the spleen. Acute tuberculosis was still les; closely simulated, and the issue of the case decided against the possibility, even had there been any doubt.

City Orthop Ædid Hospital.-The forty-sixth anniversary festival dinner in aid of the fands of this institution was held on June (30th at the Holborn Restaurant. The chairman, Baron Ferdinand de Rothschild, in proposing the toast of the evening, "The City Orthopædic Hospital," said the institution was face to face with the fact that it had to renew a mortgage of $£ 6000$, the committee having acquired the freehold of the hospital; they had, further, to appeal for funds to enable them to open additional wards. During the forty-seven years that the hospital had been in existence it had relieved upwards of 70,000 indoor and outdoor patients. It was also a free hospital and one that specially treated children. Subscriptions and donations were announced amounting to $£ 1400$ including 50 guineas from the chairman, 100 guineas from Baroness Hirsch, and 50 guineas from the City Corporation. 


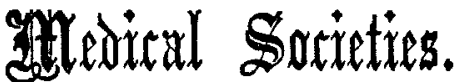

\section{OPHTHALMOLOGICAL SOCIETY.}

Spontaneous Dislocation of the Lens into the Anterior Chamber.-Demonstration of Tscherning's Theory of Accommodation. - Microphthalmos with Cystic Protrusion from the Globe.-Tuberculosis of the Conjunctiva.-Exhibition of Specimens and Cases.

A MeEting of this society was held on July 2nd, Mr. E. NETTLESHIP, President, being in the chair.

Mr. Bickenton read notes of two cases of Spontaneous Dislocation of the Lens into the Anterior Chamber. In the first case (a woman, aged sixty-five years) the right eye had been lost by an injury twenty-five years before. The sight of the remaining eye failed suddenly one afternoon without any injury being received. She was not seen till seventeen days later, when there was raised tension, vision nil, the anterior chamber being occupied by a partly opaque lens. The lens was removed without difficulty by a vectis through a corneal incision. In the second case (a man, aged sixty-six years) cataract extraction had been performed on the left eye in 1891, a preliminary iridectomy being done on the right at the same time. In March, 1897, the lens was found filling the anterior chamber of the right eye, no injury having been received. Tension was +3 , and vision equalled doubtful perception of light. The cataractous lens was removed through a corneal incision; the cortex was fluid. The vision with correction after eighty-one days was $\frac{6}{36}$.

Mr. PRIESTLter SMITH showed a model illustrating Tscherning's Theory of Accommodation, and reviewed the evidence for and against the theory. Helmholtz proved an increase of lens convexity in the area of the undilated pupil; he inferred a general increase of sphericity due to slackening of the zonula. He put forward his theory as provisionally the most feasible, not as proved. Tscherning, following Thomas Young, shows that during accommodation the refraction and the convexity of the lens increase chiefly at the central area of the pupil, less and less towards the periphery, and in the peripheral zones not at all; the lens surface changes from a spherical to a hyperboloid curvature. $\mathrm{He}$ shows further that this change can be produced in animals by tightening the zonula. Tscherning's observations relate to:-1. Spherical aberration: Did the sphericity of the lens increase during accommodation, the spherical aberration, other things being equal, would increase also. But a study of diffusion circles and shadows cast upon the retina shows no such increase, but the reverse; the peripheral zones of the lens do not increase in refraction as does the central area. 2 . Scheiner's experiment: Looking at a point of light through two adjacent pinholes placed before the pupil, the eye sees it single at the distance for which it is focussed and double at any greater or less distance. With an instrument based on this principle the refraction is estimated in different parts of the pupillary area at the same time. During accommodation the increase of refraction, measured in this way, is much greater at the centre than at the periphery. 3. Reflection from the lens surface in the living eye: A series of electric lamps placed in a horizontal line before the eye are seen reflected by the lens. The varying degrees of displacement which these reflections undergo during accommodation are an index to the change of curvature in the lens surface. The convexity increases chiefly at the centre, less and less, or not at all towards the periphery. 4. Reflection from the surface of the exposed lens and traction of the zonula: The zonula being seized and rendered tense at two opposite points of the circle, the change of curvature occurring in the lens surface is studied by means of images reflected from it in several zones. It is of the character already described. Tscherning holds, therefore, that in the act of accommodation the ciliary muscle contracts so as to increase the tension of the zonula, and to alter the lens surface from a spherical to a hyperboloid form. A given increase of convexity in the central area involves much less change the general form of the lens than it would according to the assumption of Helmholtz. Tscherning founds his study on the work of Thomas Young, and does full honour to that eminent man of science. Hess, on the other hand, shows that during strong contraction of the ciliary muscle, especially under eserine, the lens becomes tremulous, and this he declares is incompatible with an increased tension of the zonula. Tscherning confirms the observation, but attributes the trembling of the lens to spasmodic action of the ciliary muscle. Hess replies that the lens, besides being tremulous, is displaced laterally, the displacement in all positions of the head being governed by gravity. This, he urges, can only be explained by assuming that contraction of the ciliary muscle slackens the zonula.

Mr. Treacher Coldins and Mr. J. R. Rolston read a paper on Microphthalmos with Cystic Protrusion from the Globe, based on the pathological examination of seren eyes in which it was present. It was pointed out that though all the specimens were alike in one particular-namely, in being microphthalmic eyes with a protrusion of retinal tissue through a gap in the choroid and sclerotic-they presented great differences as regards the degree of development of the eyeball. The amount of the protrasion of retina varied from a small knuckle of tissue to quite two-thirds of that membrane. In some of the cases the folds of retina had become distended into one or more fluids containing cysts. The region of the globe in which the protrusions were situated was nearly always its lower and posterior part. Speaking generally, the larger the amount of the retinal protrusion the smaller and more imperfectly developed was. the eyeball. In some of them the vitreous was rery imperfectly formed, being replaced by a kind of fibrous tissue with blood-vessels coursing through it. This so-called "atypical development of the vitreous humour," in which the mesoblast which should have developed into vitreous. humour had instead developed into fibrous tissue, was thought to be a matter of considerable importance, as it served to elucidate cases of congenital amblyopia, which when examined ophthalmoscopically were seen to have fibrous membranes or bands in the vitreous chamber. In one of the specimens they had found a large plate of welldeveloped hyaline cartilage embedded partly in the sclerotic and partly in the cornea. They referred to two other some what similar cases recorded by Lapersonne and Mitvalsky in which nodules of hyaline cartilage had also been found in the sclerotic. It was thought the changes in all their cases could be best explained by supposing the retina, through imperfect development of the vitreous or delayed closure of the ocalar cleft, to have become much folded, and that one or more of these folds had become extended through the ocular cleft into the subjacent mesoblast, where in some of the cases it had subsequently expanded into fluid-containing cysts. The paper was fully illustrated by lantern slides.

Dr. J. FYRe read a paper on Tuberculosis of the Conjunctiva. According to his statistics the frequency of these cases was about 1 in 3000 . They were divided into five groups-the first characterised by miliary ulceration of the conjunctiva, the second by sub.conjunctival nodules somewhat resembling trachoma granules, the third by superabundant papillary granulations, the fourth appeared as lupus of the conjunctiva, and the fifth as papillomatous tumours of the palpebral conjunctiva. The affection was to be regarded as the result of direct inoculation and not as an expression of general tuberculosis. The age limit varied from ten montbs to thirty years, the most common time of appearance being about puberty; females were more often attacked than males. The conjunctiva of the lower lid was the most common seat of the affection. glandular enlargement was generally present. Eight cases were described in detail. In group 1, 1 case; group 2, 2 cases; group 3,2 cases; group 4, 1 case; and group 5, 2 cases. Special attention was directed to group 5 on account of the difficulty in diagnosis without microscopic examination. The necessity of early complete removal of the disease was insisted on.

The following cases and card specimens were shown :-

Mr. JESSOP : Sarcoma of Choroid.

Dr. BATten : Case of Spontaneous Hæmorrhage into the Vitreous.

Mr. J. GRiffith : Case of Probable Thrombosis of the Cavernous Sinus.

Mr. Holmes Spicer and Mr. DevereuX Marshall: Specimen of Tubercle of the Tris.

Mr. Thомpson : Unusual Type of Corneal Infiltration. 